\title{
Autism Spectrum Disorders: Five Things You Should Know about Autism Treatment
}

\author{
Renee L. Bauer \\ Department of Baccalaureate Nursing, College of Health and Human Services, Indiana State University, USA
}

Copyright $\bigcirc 2016$ by authors, all rights reserved. Authors agree that this article remains permanently open access under the terms of the Creative Commons Attribution License 4.0 International License

\begin{abstract}
Autism spectrum disorders (ASDs) are typically very complex neurobiological disabilities that concur with developmental disabilities. Generally, this illness presents during the first three years of life and is more commonly found in males. Ranging from mild, moderate, to severe, this disease affects the normal development of the brain regarding social interactions and communication skills (Halter, 2014). Symptoms present as mild, such as the inability to bond with others, to aggression such as head banging and rage. Characteristically, individuals display delayed age appropriate interactions and require intensive intervention if they are to lead a productive life. The prevalence of autism is on the upsurge and with this comes the recent revision of the American Psychiatry Associations' taxonomy (DSM-V) (Brady, 2014). Both genetics and the environment may play a role in causes of this disorder (NINDS, 2014). Between 20 to 30 percent of children with ASD develop epilepsy by the time they are an adult. Not unlike other disorders, autism does have a genetic link and many children with autism have a relative with the disease. The controversy surrounding autism with the measles, mumps and rubella (MMR) vaccination is still continuing. Many individuals felt that this vaccination lead to the late development of the late-onset disorder. The National Institute of Child Health and Human Development, Center for Disease Control (CDC) and the Academy of Pediatrics have been conducting research for years and made a conclusion as there is no link to MMR and the development of the disease (Videbeck, 2014).
\end{abstract}

Keywords Autism, Applied Behavior Analysis, Increased Diagnosis, Early Recognition

\section{Introduction}

Autism spectrum disorders (ASDs) are typically very complex neurobiological disabilities that concur with developmental disabilities. Generally, this illness presents during the first three years of life and is more commonly found in males. Ranging from mild, moderate, to severe, this disease affects the normal development of the brain regarding social interactions and communication skills (Halter, 2014). Symptoms present as mild, such as the inability to bond with others, to aggression such as head banging and rage. Characteristically, individuals display delayed age appropriate interactions and require intensive intervention if they are to lead a productive life.

The prevalence of autism is on the upsurge and with this comes the recent revision of the American Psychiatry Associations' taxonomy (DSM-V) (Brady, 2014). Both genetics and the environment may play a role in causes of this disorder (NINDS, 2014). Furthermore, between 20 to 30 percent of children with ASD develop epilepsy by the time they are an adult.

Not unlike other disorders, autism does have a genetic link and many children with autism have a relative with the disease. The controversy surrounding autism with the measles, mumps and rubella (MMR) vaccination is still continuing. Many individuals felt that this vaccination lead to the late development of the late-onset disorder. The National Institute of Child Health and Human Development, Center for Disease Control (CDC) and the Academy of Pediatrics have been conducting research for years and made a conclusion as there is no link to MMR and the development of the disease (Videbeck, 2014).

\section{Early Diagnosis}

Common signs of autism may be the child fails to respond to their name and is frequently seen avoiding eye contact (NINDS, 2014). Impaired social interaction is one of the hallmark features of the disorder. Frequently, children display repetitive movements such as rocking or head-banging. Speech is often delayed. Lacking empathy is another common characteristic. Autism may go unrecognized in mildly affected children.

Early indicators include:

- No pointing or babbling by age 1

- No single words by 16 months

- No response to name

- Loss of language or lack of social cues 
- Lack of eye contact

- Lining up of toys or objects on a frequent basis

- No smiling and lack of social responsiveness

Later indicators include:

- Inability to make friends

- Inability to sustain conversations with others

- Repetitive or unusual use of language

- Inflexible adherence to routine

\section{Prompt Intervention}

Like most psychiatric illnesses, early intervention is crucial is assisting children to lead productive lives. In the journal of Pediatrics, the official journal of the American Academy of Pediatrics (AAP), noted, "Children who receive early intensive behavioral treatment have been shown to make substantial, sustained gains in IQ, language, academic performance, and adaptive behavior as well as some measures of social group, and their outcomes have been significantly better than those of children in control groups" (Myers \& Johnson, 2007, p. 1164). Although there is no cure, specific treatments are targeted to remedy symptoms and enhance improvement (NINDS, 2014). Ideal treatment plans coordinate therapies and align interventions that tailor to the needs of the individual children.

Currently, therapists use highly structured and time intensive skill oriented training. This is known as Applied Behavior Analysis (ABA), once commonly called behavior modification (NINDS, 2014). Comprehensive ABA interventions are aimed at producing changes in specific skills impacting global measures. These maybe are IQ, adaptive skills, and social functioning (Hagopian \& Hardesty, 2014). Usually treatment lasts over an extended period of time and is home centered or treatment base centered. It is not unusual to have programming ranging from 25-40 hours per week. Some of these treatments may last up to two years.

The Harsha Cognitive Center recently opened spring 2014 in Terre Haute Indiana and is seeing children from ages three to 22 years of age. Skills are taught that include, socialization, attention, discrimination and language communication. These programs rely on the use of clear instruction, reinforcement, teaching small units of behavior, and repeated learning trials. When this intervention is used with younger children it is often called "early intensive behavioral intervention" (EIBI) (Hagopian \& Hardesty, 2014). Employees are called "coaches" and are 1:1 with children. Staffs see improvements and track the progress of each child on a daily basis. Rewards are commonly M\&Ms and high fives. Or, rewarded behavior may consist of the ability to play with a favored toy or game. All treatment is individualized and developed for the one patient receiving the treatment. All ABA behavior used at the center is evidence based and followed on a daily basis. Recently, the center was able to work successfully with a male who was 22 and had not been able to be toilet trained. After several months, he was making improvement in this area. His parents were very grateful and appreciative. They saw the gains in a relatively short period of time (one year) and continue to praise the center.

Staffs who work at the center report increased satisfaction from seeing patients improve. They bond with their patients. Many caregivers take ownership in their roles as ABA specialists. Families are routinely satisfied with the patient progress. Ultimately, this leads to a positive dynamic with all involved. Frequently those who work on an inpatient unit do not see the same kind of progress or do not get to bond with patients due to shortened length of stay.

Many research studies have demonstrated that center-based comprehensive ABA interventions are highly effective in improving IQ, adaptive skills, and social performance when programming is supplied 25-40 hours per week. Nearly four decades of research demonstrated that ABA-based treatment approaches are effective in problem reduction. Features common to ABA-based therapy approaches are: 1) the objective measurement of behavior, 2) Use of procedures based on scientifically established principles of behavior, and 3) a precise control of the environment to allow for the objective evaluation of outcomes (Hagopian \& Hardesty, 2014).

Over a thousand studies reporting on ABA-based assessment have been published since the 1960s (Hagopian \& Hardesty, 2014). Literature consists of numerous controlled studies using single-cased experimental designs, consecutive controlled case-series studies, controlled group studies, and some randomized control trials. Hagopian, Fisher, Sullivan, Acquisto, \& LeBLanc (1998) conducted large-scale consecutive controlled case studies providing effectiveness of interventions. Findings from large studies parallel those that are smaller and replicate meta-analysis of small-n studies.

\section{Medication Administration}

Pharmacological agents are commonly used for agitation, compulsive behaviors, or anxiety (Halter, 2014). The reduction of harmful behaviors may be accomplished with the use of the newer unconventional agents; antipsychotics assist with controlling severe behavioral problems (NINDS, 2014). Medications such as haloperidol (Haldol) or risperidone (Risperdal) are effective in aggression (Videbeck, 2014). The SSRIs are more commonly used to reduce anxiety and enhance mood.

\section{Parental Involvement}

Often parents need assistance when it comes to gearing their child and facilitating their child to lead a productive life. Taking the child to a treatment center allows for a respite. Parents come back refreshed while children are looked after. Families usually are optimistic when they see their child 
improve after living years with minor developmental milestones met.

Parents must understand developmental milestones such as adolescence may cause deterioration in behavior. Hormonal changes along with the stress of meeting social demands can be a challenge in this population (Videbeck, 2014). Social skills rarely improve to the degree that patients are totally independent of assistance. Adults with autism may be viewed as odd or are inaccurately diagnosed to have a schizoid personality.

Parenting a child with autism distresses both cognitive and affective domains of the parents (Serrata, 2012). Research suggests that stress levels and depression impede healthy family relationships and take a toll on financial stability due to the high cost in treatment. Parents go through grieving upon diagnosis of autism and often times it is the mother who accepts the caregiver role and reports caring for their child is a full time responsibility. Rehabilitation counselors are vital in order to provide the parents with educational and social support.

Social networking and social support are vital when a child is diagnosed with autism (Serrata, 2012). A study conducted by Twoy et al. (2007) illustrated the various supports that parents use. Investigators found that $68 \%$ of parents gained support from their friends, $58 \%$ received support from close family relatives, and $47 \%$ received support from spiritual guidance. A large majority, as much as $93 \%$, also gained strength in others who had children with a similar diagnosis (Serrata, 2012).

There are several resources on the internet the parents may utilize. The Autism Society of America, Autism Speaks, the Interactive Autism Network, Unlocking Autism, and the National Dissemination Center for Children with Disabilities are internet resources that provide counseling and information. It is documented that parents receiving ongoing professional and educational support benefit the best (Serrata, 2012).

\section{Realistic Outcomes}

Parents, and or, care givers need to set realistic expectations. Those with ADS take small steps toward making improvements. Nurses and other healthcare workers may suggest support groups to aid with parental assistance. Autism traits persist into adulthood and parents must be patient with making progress (Videbeck, 2014).

Luckily, many insurance coverage laws are initiating widespread insurance reform leading implementation of
ABA services. Recently, military children are receiving treatment for medical necessity rather than educational services. Since 2007, more than half of the United States using insurance reform is successful in passing bills which require insurance companies to pay for autism treatment mainly consisting of ABA services (Hagopian \& Hardesty, 2014).

Early intervention and successful planning can alleviate some of the obstacles parents face when they have a child diagnosed with autism. Unfortunately, more children are being diagnosed with this disorder but there is hope. Parents benefit when they are proactive in using all available tools.

\section{REFERENCES}

[1] Autism Fact Sheet (2014). NINDS, NIH Publication No. 09-1877

[2] Brady, L.J. (2014). Lois Jean Brady: Apps for Autism: An essential guide to over 200 effective apps for improving communication, behavior, social skills, and more! Journal of Autism and Developmental Disorders, 44(9). doi: 10.1007/s10803-014-2134-6

[3] Hagopian, L.P., \& Hardesty, S.L. (March, 2014). Applied Behavior Analysis: Overview and Summary of Scientific Support. KennedyKrieger.org. Retrieved September 27, 2014 fromhttp://www.kennedykrieger.org/patient-care/patient-car e-programs/inpatient-programs/neurobehavioral-unit-nbu/ap plied-behavior-analysis

[4] Hagopian, L. Fisher, W., Sullivan, M., Acquisto, J., \& LeBlanc, L. (1998). Effectiveness of functional communication training with and without extinction and punishment: A summary of 21 inpatient cases. Journal of Applied Behavior Analysis, 31,211-235.

[5] Halter, M.J. (2014). Varcarolis' Foundations of Psychiatric Mental Health Nursing: A Clinical Approach. St. Louis, MO: Elsevier

[6] Myers, S., \& Johnson, C. (2007). Management of children with autism spectrum disorders. Pediatrics, 120, 1162-1182.

[7] Serrata, C. (2012). Psychosocial aspects of parenting a child with autism. Journal of Applied Rehabilitation and Counseling, 43(4) 29-35.

[8] Twoy, R.,Connolly,P. M.,\& Novak, J.M. (2007) Coping strategies used by parents with autism. Journal of the Academy of Nurse Practitioners, 19,251-260. doi: $10.1177 / 0034355208324263$

[9] Videbeck, V. (2014). Psychiatric Mental Health Nursing. Philadelphia, PA: Lippincott Williams \& Wilkins 